\title{
Fatores associados a agravos gastrointestinais no primeiro ano de prematuros tardios e moderados
}

\author{
Factors associated with gastrointestinal disorders in the first year of late and moderate \\ premature infants
}

\section{Como citar este artigo:}

Jantsch LB, Barzotto VS, Silva EB. Factors associated with gastrointestinal disorders in the first year of late and moderate premature infants. Rev Rene. 2020;21:e43243. DOI: https://doi.org/10.15253/2175-6783.20202143243

\author{
DLeonardo Bigolin Jantsch ${ }^{1}$ \\ (1) Vanessa da Silva Barzotto ${ }^{1}$ \\ (1DEthel Bastos da Silva ${ }^{1}$
}

${ }^{1}$ Universidade Federal de Santa Maria.

Palmeira das Missões, RS, Brasil.

\section{Autor correspondente:}

Leonardo Bigolin Jantsch

Av Independência, 3751, Vista Alegre -

CEP: 98300-000. Palmeira das Missões, RS, Brasil.

E-mail: leo_jantsch@hotmail.com

\begin{abstract}
RESUMO
Objetivo: analisar os fatores associados ao desenvolvimento de agravos gastrointestinais, no primeiro ano de vida de prematuros tardios e moderados. Métodos: estudo longitudinal, que acompanhou lactentes nascidos tardios e moderados, durante o primeiro ano de vida, via telefone, para desenvolvimento de agravos gastrointestinais, na percepção materna. Dados coletados por meio de escala likert, na avaliação da condição aguda, e analisados sob comparação de frequência e odds ratio $(\mathrm{p}<5 \%$ e Intervalo de Confiança 95\%) entre as variáveis. Resultados: os agravos gastrointestinais mais frequentes na avaliação dos primeiros meses de vida foram a cólica e o vômito, que reduziram a intensidade, quanto mais perto do primeiro ano de vida do lactente, seguidos do agravo diarreia que manteve intensidade constante ao longo do primeiro ano. Conclusão: o estudo traz implicação para identificação dos fatores de risco, tendo em vista a vulnerabilidade da população de prematuros, ainda pouco descrita na literatura.

Descritores: Vômito; Cólica; Diarreia; Doenças do Prematuro; Fatores de Risco.
\end{abstract}

\section{ABSTRACT}

Objective: to analyze the factors associated with the development of gastrointestinal disorders in the first year of life of late and moderate premature infants. Methods: longitudinal study, which followed infants born late and moderate, during the first year of life, via telephone, to develop gastrointestinal disorders, in the maternal perception. Data collected using the Likert scale, in the assessment of acute condition, and analyzed under comparison of frequency and odds ratios ( $\mathrm{p}<5 \%$ and 95\% Confidence Interval) between variables. Results: the most frequent gastrointestinal injuries in the assessment of the first months of life were colic and vomiting, which reduced the intensity, the closer to the infant's first year of life, followed by the diarrheal condition that maintained constant intensity throughout the first year. Conclusion: the study has implications for the identification of risk factors, in view of the vulnerability of the premature population, still little described in the literature.

Descriptors: Vomiting; Colic; Diarrhea; Infant, Premature, Diseases; Risk Factors. 


\section{Introdução}

A Organização Mundial da Saúde classifica prematuro tardio e moderado aquele recém-nascido com idade gestacional de 32 a 36 semanas e seis dias ${ }^{(1)}$. Esses prematuros devem ser considerados de alto risco, pois a prematuridade os tornam instáveis fisiológica e hemodinamicamente, com risco para distúrbios congênitos, alterações metabólicas e asfixia perinatal, necessitando de cuidados especiais ${ }^{(2)}$.

A elevada taxa de prematuridade no Brasil é predominante entre os prematuros tardios, na maioria das vezes, ocorre de forma espontânea, porém, ainda se vivenciam taxas de cesarianas iatrogênicas, as quais intensificam os números de prematuridade, que atualmente giram em torno de $11,0 \%$ na população geral, destes, 88,0\% são considerados prematuros com mais de 32 semanas de idade gestacional ${ }^{(3)}$.

Reconhece-se que prematuros tardios e moderados apresentam imaturidade nos sistemas de regulação fisiológica. Ao considerar essa característica, destacam-se aspectos da imaturidade gastrointestinal, em que manifestação como vômito, refluxo, diarreia e cólica podem estar mais frequentes. Por volta da sétima semana de gestação, o estômago está anatomicamente maduro, porém as contrações rítmicas vão ocorrer a partir de quatro dias de vida do recém-nascido, aproximadamente. Em prematuros, esse esvaziamento é mais lento e pode ser mais um motivo para maior intensidade da cólica, justificado também pela imaturidade transitória das funções intestinais, como as funções entero-hepáticas e a homeostase dos ácidos biliares, a motilidade gastrointestinal e o microbiota do cólon ${ }^{(4)}$.

As repercussões gastrointestinais em prematuros não se estabelecem apenas no período neonatal, visto que na infância desses prematuros, os distúrbios gastrointestinais são muito prevalentes, ora funcionais, ora estruturais. Os funcionais não apresentam anormalidade e/ou deformações anatômicas. Nas estruturais, manifestam-se de forma determinante. Contudo, vários fatores podem estar associados ao quadro clínico ${ }^{(5)}$. Alergias alimentares, como esofagite eosinofílica, doença celíaca, distúrbios metabólicos, intolerâncias alimentares são afecções que podem estar relacionadas às condições digestórias, e os períodos curtos e transitórios, ainda, mais desafiadores ${ }^{(6-7)}$. Já a doença de refluxo gastroesofágico, considerada a enfermidade esofágica mais comum no período neonatal, ainda apresenta aspectos e definições não adequadamente conhecidos, principalmente em prematuros tardios e a termos ${ }^{(8-9)}$.

Existe número elevado de lactentes que apresentam sinais e sintomas gastrintestinais. Parcela desses lactentes expõe mais de um sintoma e essas manifestações frequentemente são motivo de mudanças no esquema alimentar do lactente, principalmente, mudanças nos tipos de fórmulas infantis, o que pode repercutir nos aspectos de crescimento e desenvolvimento infantil e fatores de risco para agravos futu$\operatorname{ros}^{(10)}$.

Para tanto, discutir sobre a ocorrência desses agravos e fatores associados em uma população considerada "quase a termo", em serviços da atenção à saúde, prematuros moderados e tardios, é extremamente relevante, tendo em vista quadro de ascensão epidemiológica desses bebês e manifestações das funções biológicas imaturas. Assim, o reconhecimento dos fatores permite identificação precoce e orientação dos serviços, a fim de minimizar os agravos. Deste modo, este estudo teve como pergunta norteadora: quais os fatores neonatais e socioeconômicos associados à intensidade de agravos gastrointestinais de prematuros tardios e moderados no primeiro ano de vida? Para responder à pergunta de estudo, objetivou-se analisar os fatores associados ao desenvolvimento de agravos gastrointestinais, no primeiro ano de vida de prematuros tardios e moderados.

\section{Métodos}

Estudo longitudinal aberto, oriundo do banco de dados do projeto intitulado Condições de saúde de prematuros moderados e tardios no primeiro ano de 
vida, desenvolvido no município de Santa Maria, RS, Brasil.

A seleção dos participantes foi realizada durante um ano de coleta de dados (maio/2017 a maio/2018), com objetivo de reduzir viés de sazonalidade (região do estudo com condições climáticas em que extremos de temperaturas -inverno e verãopudessem interferir nos agravos à saúde), em Centro Obstétrico de hospital de referência para gestação de alto risco para região central do Rio Grande do Sul. Após a seleção dos participantes, ocorreu a busca ativa destes nos serviços de Alojamento Conjunto (Unidade Tocoginecológica) e Unidade de Terapia Intensiva Neonatal. 0 acompanhamento e a coleta dos dados aconteceram por meio de contato telefônico dos pesquisadores, no primeiro mês de vida. A equipe de coleta de dados, que acompanhou todas as etapas, foi composta por um pesquisador responsável e auxiliares de pesquisa, graduandos da área da saúde.

Os participantes do estudo foram 151 recém-nascidos prematuros, que nasceram com idade gestacional, no período de 32 a 36 semanas e seis dias. 0 grupo foi classificado em prematuros tardios, aqueles que nascerem com 34 a 36 semanas e seis dias de idade gestacional; e prematuros moderados aquele que tiveram de 32 a 33 e seis dias. Para seleção inicial dos participantes, a idade gestacional foi considerada aquela apresentada no livro de registro de nascimentos do hospital e no prontuário do paciente, determinada pelo método de Capurro, pós-neonatal imediato.

Como critérios de seleção, incluíram-se no estudo os recém-nascidos considerados prematuros moderados ou tardios, nascidos na instituição em que foi realizado o estudo, e residentes em Santa Maria, no momento da alta hospitalar. Utilizou-se de formulário próprio, devidamente testado, cujas variáveis neonatais e socioeconômicas foram coletadas durante a internação. As variáveis neonatais foram: idade gestacional (moderado e tardio), peso ao nascer (PIG= Pequeno para Idade Gestacional, $\mathrm{AIG}=$ Adequado para Idade Gestacional, GIG= Grande para Idade Gestacional), necessidade de internação em Unidade Terapia
Intensiva Neonatal) ou alojamento conjunto e tipo de alimentação. Quanto à classe socioeconômica, os grupos foram alocados em classes dos tipos A-B, C e D-E.

$\mathrm{O}$ contato para acompanhamento foi realizado nos terceiro, sexto e $12^{\circ}$ meses de vida, para viabilidade do estudo; e para diminuição dos gastos com transporte, pelo telefone. Os meses de avaliação foram definidos conforme a frequência estabelecida pelo instrumento de avaliação, de forma trimestral. 0 instrumento caracteriza-se como do tipo Likert, em que as condições de saúde (cólica, vômito e diarreia) são avaliadas e classificadas conforme os escores. Avaliaram-se vômito e diarreia, nas apreciações de terceiro e $12^{\circ}$ meses; a condição cólica foi avaliada nos $3^{\circ} \mathrm{e}$ 6 o meses de vida. As respostas variavam de nunca $=0$, quase nunca $=1$, quase sempre $=2$ e sempre $=3^{(11)} .0$ instrumento foi traduzido, adaptado e validado para português no ano de 2010. Por entender que as condições agudas possuem repercussões, em especial aos cuidadores, quem definem a intensidade do agravo e o quanto essa situação possa vir a repercutir para saúde da criança e procura dos serviços de saúde.

Quanto à análise das variáveis associadas à frequência dos agravos agudos, aplicaram-se os testes de comparação de frequência (empregando o teste Qui-Quadrado), utilizando-se do programa estatístico software Statistical Package for the Social Sciences for Windows, versão 20.0. Estabeleceu-se associação estatística significativa ao desfecho, às variáveis cujo valor $\mathrm{p}$ foi menor ou igual a $5 \%(\mathrm{p} \leq 0,05)$. Na análise de Odds Ratio (OR) (razão de chance) das variáveis vômito e cólica, adotaram-se as comparações das respostas Nunca e Sempre e Intervalo de Confiança (IC) de 95\%. $\mathrm{Na}$ análise da OR da variável diarreia, utilizaram-se das respostas Nunca e Quase Sempre para cálculo e IC95\%, visto que a resposta Sempre apresentou baixa frequência $(n=1)$.

O estudou seguiu as recomendações éticas e foi aprovado pelo Parecer Consubstanciado do Comitê de Ética em Pesquisa da Universidade Federal de Santa Maria, no 1.511.201/2016, e Certificado de Apresentação para Apreciação Ética no 53898916.9.0000.5346. 


\section{Resultados}

Participaram do estudo na avaliação do terceiro mês 119 crianças que foram avaliadas quanto ao vômito, à cólica e diarreia [21,2\% de perda (1 perda por óbito; 31 por ausência de resposta por contato telefônico)]; na avaliação do sexto mês, avaliaram-se 108 crianças somente para o agravo cólica [perda de $28,5 \%$ ( houve mais cinco perdas por ausência de resposta por contato telefônico; seis por desistência em participar do estudo)]; e, na última avaliação, aos 12 meses de idade, quanto aos agravos vômito e diarreia, participaram 105 crianças, com perda de 30,0\% da população inicial (mais três perdas por desistência em participar do estudo).
Na Tabela 1, estão apresentadas a intensidade do agravo vômito e a comparação das variáveis com a intensidade, nos terceiro e $12^{\circ}$ meses de vida.

Na análise das variáveis neonatais e socioeconômicas, não se constatou diferença significativa entre a intensidade do vômito nos terceiro e $12^{\circ}$ meses de vida. Destaca-se que, no terceiro mês, 8,3\% dos bebês que não receberam aleitamento materno apresentaram vômito sempre, comparado aos $4,7 \%$ daqueles que estavam em aleitamento materno exclusivo. Ainda, as classes socioeconômicas C, D-E apresentaram maior frequência da intensidade, sempre, quando comparadas as demais classes, $13,0 \%$ mais chances de desenvolver vômito no terceiro mês de vida. $\mathrm{Na}$ Tabela 2, destaca-se o agravo diarreia, na comparação com as variáveis.

Tabela 1 - Distribuição da intensidade do agravo vômito, nos terceiro e 12을 meses de vida de lactentes prematuros tardios e moderados. Santa Maria, RS, Brasil, 2019

\begin{tabular}{|c|c|c|c|c|c|}
\hline \multirow{2}{*}{ Variáveis neonatais e socioeconômicas } & Nunca & Sempre & \multirow{2}{*}{$\mathbf{p}^{*}$} & \multirow{2}{*}{ Odds Ratio } & \multirow{2}{*}{ IC $+95 \%$} \\
\hline & n(\%) & n(\%) & & & \\
\hline Terceiro mês & $57(47,1)$ & $5(5,0)$ & \multirow{4}{*}{0,531} & \multirow{5}{*}{0,71} & \multirow{5}{*}{$0,07-7,14$} \\
\hline Moderado & $7(38,9)$ & $1(5,6)$ & & & \\
\hline Tardio & $50(49,5)$ & $5(5,0)$ & & & \\
\hline Pequeno para idade gestacional & $11(45,8)$ & $1(4,2)$ & & & \\
\hline Adequado para idade gestacional & $42(47,2)$ & $5(5,6)$ & \multirow[t]{2}{*}{0,499} & & \\
\hline Grande para idade gestacional & $4(66,7)$ & - & & \multirow{3}{*}{1,2} & \multirow{3}{*}{$0,202-7,12$} \\
\hline Egresso da terapia intensiva & $21(43,8)$ & $2(4,2)$ & \multirow{2}{*}{0,412} & & \\
\hline Alojamento conjunto & $36(50,7)$ & $4(5,6)$ & & & \\
\hline Aleitamento materno & $53(49,5)$ & $5(4,7)$ & \multirow{2}{*}{0,480} & \multirow[t]{2}{*}{2,6} & \multirow[t]{2}{*}{$0,246-27,97$} \\
\hline Não recebeu aleitamento materno & $4(33,3)$ & $1(8,3)$ & & & \\
\hline A-B & $12(57,1)$ & - & \multirow{2}{*}{0,201} & \multirow{3}{*}{1,13} & \multirow{3}{*}{$1,026-1,259$} \\
\hline C, D-E & $44(34,1)$ & $6(4,6)$ & & & \\
\hline $12^{\circ}$ mês & $74(70,5)$ & $1(1,0)$ & & & \\
\hline Moderado & $12(70,6)$ & - & \multirow{2}{*}{0,559} & \multirow[t]{2}{*}{1,01} & \multirow[t]{2}{*}{$0,98-1,05$} \\
\hline Tardio & $62(70,5)$ & $1(1,1)$ & & & \\
\hline Pequeno para idade gestacional & $16(72,7)$ & - & \multirow{3}{*}{0,917} & & \\
\hline Adequado para idade gestacional & $54(70,1)$ & $1(1,3)$ & & & \\
\hline Grande para idade gestacional & $4(66,7)$ & - & & & \\
\hline Egresso da terapia intensiva & $29(69,0)$ & - & \multirow{2}{*}{0,258} & 1,02 & \multirow[t]{2}{*}{$0,97-1,06$} \\
\hline Alojamento conjunto & $45(71,4)$ & $1(1,6)$ & & & \\
\hline Aleitamento materno exclusivo & $70(72,2)$ & $1(1,0)$ & \multirow{2}{*}{0,231} & \multirow[t]{2}{*}{0,98} & \multirow[t]{2}{*}{$0,95-1,01$} \\
\hline Não recebeu aleitamento materno & $4(50,0)$ & - & & & \\
\hline A-B & $15(75,0)$ & - & \multirow{2}{*}{0,615} & \multirow[t]{2}{*}{1,01} & \multirow[t]{2}{*}{$0,98-1,05$} \\
\hline C-D-E & $59(69,4)$ & $1(1,6)$ & & & \\
\hline
\end{tabular}


Tabela 2 - Distribuição da intensidade do agravo diarreia, nos terceiro e $12^{\circ}$ meses de vida de lactentes prematuros moderados e tardios. Santa Maria, RS, Brasil, 2019

\begin{tabular}{|c|c|c|c|c|c|}
\hline Variáveis neonatais e socioeconômicas & $\begin{array}{l}\text { Nunca } \\
\text { n(\%) } \\
\end{array}$ & $\frac{\text { Quase Sempre }}{\mathrm{n}(\%)}$ & $\mathbf{p}^{*}$ & Odds Ratio & $\mathrm{IC}^{\dagger} \mathbf{9 5 \%}$ \\
\hline Terceiro mês & $86(72,3)$ & $8(6,7)$ & \multirow{4}{*}{0,629} & \multirow{5}{*}{1,11} & \multirow{5}{*}{$1,03-1,19$} \\
\hline Moderado & $14(77,8)$ & 0 & & & \\
\hline Tardio & $72(71,3)$ & $8(7,9)$ & & & \\
\hline Pequeno para idade gestacional & $15(62,5)$ & $1(4,2)$ & & & \\
\hline Adequado para idade gestacional & $65(73,0)$ & $7(7,9)$ & \multirow[t]{2}{*}{0,241} & & \\
\hline Grande para idade gestacional & $6(100,0)$ & 0 & & \multirow{3}{*}{0,61} & \multirow{3}{*}{$0,13-2,88$} \\
\hline Egresso da terapia intensiva & $31(64,6)$ & $2(4,2)$ & \multirow{2}{*}{0,105} & & \\
\hline Alojamento conjunto & $55(77,5)$ & $6(8,5)$ & & & \\
\hline Aleitamento materno & $80(74,8)$ & $7(6,5)$ & \multirow{2}{*}{0,012} & \multirow{3}{*}{0,56} & \multirow{3}{*}{$0,08-3,95$} \\
\hline Não recebeu aleitamento materno & $6(50,0)$ & $1(8,3)$ & & & \\
\hline A-B & $13(59,1)$ & $2(9,1)$ & \multirow{3}{*}{0,314} & & \\
\hline C, D-E & $73(56,6)$ & $6(27,2)$ & & 1,9 & \multirow{2}{*}{$0,42-8,5$} \\
\hline $12^{\circ}$ mês & $76(72,1)$ & $4(3,8)$ & & & \\
\hline Moderado & $12(70,6)$ & 0 & \multirow{2}{*}{0,597} & & \multirow{3}{*}{$1,01-1,13$} \\
\hline Tardio & $64(72,7)$ & $4(4,5)$ & & 1,06 & \\
\hline Pequeno para idade gestacional & $16(72,7)$ & 0 & & & \\
\hline Adequado para idade gestacional & $55(70,5)$ & $4(5,2)$ & \multirow[t]{2}{*}{0,441} & \multirow{6}{*}{0,49} & \multirow{6}{*}{$0,05-4,49$} \\
\hline Grande para idade gestacional & $6(100,0)$ & 0 & & & \\
\hline Egresso da terapia intensiva & $31(73,8)$ & $1(2,4)$ & \multirow{2}{*}{0,809} & & \\
\hline Alojamento conjunto & $45(71,4)$ & $3(4,8)$ & & & \\
\hline Aleitamento materno & $71(73,2)$ & $4(4,3)$ & \multirow{2}{*}{0,578} & & \\
\hline Não recebeu aleitamento materno & $5(62,5)$ & 0 & & & \\
\hline A-B & $15(75,0)$ & 0 & \multirow{2}{*}{0,043} & & \\
\hline C, D-E & $61(47,3)$ & $4(3,1)$ & & 1,07 & $1,01-1,36$ \\
\hline
\end{tabular}

Na análise das variáveis neonatais e socioeconômicas, percebeu-se diferença na prevalência da intensidade quase sempre da diarreia, nos terceiro e $12^{\circ}{ }^{\circ}$ meses de vida. Os bebês nascidos tardios obtiveram maior risco para desenvolver diarreia nos terceiro e $12^{\circ}$ meses de vida. Enfatiza-se que, no terceiro mês, $74,8 \%$ dos bebês que receberam aleitamento mater- no exclusivo, nunca apresentavam diarreia, comparados aos 50,0\% daqueles que estavam em aleitamento materno. Ainda, as classes socioeconômicas C, D-E indicaram maior frequência da intensidade, sempre, e maior risco para desenvolver diarreia ao final do primeiro ano de vida. Na Tabela 3, destaca-se o agravo cólica, na comparação com as variáveis.

Tabela 3 - Distribuição da intensidade do agravo cólica, nos terceiro e sexto meses de vida de lactentes prematuros moderados e tardios. Santa Maria, RS, Brasil, 2019

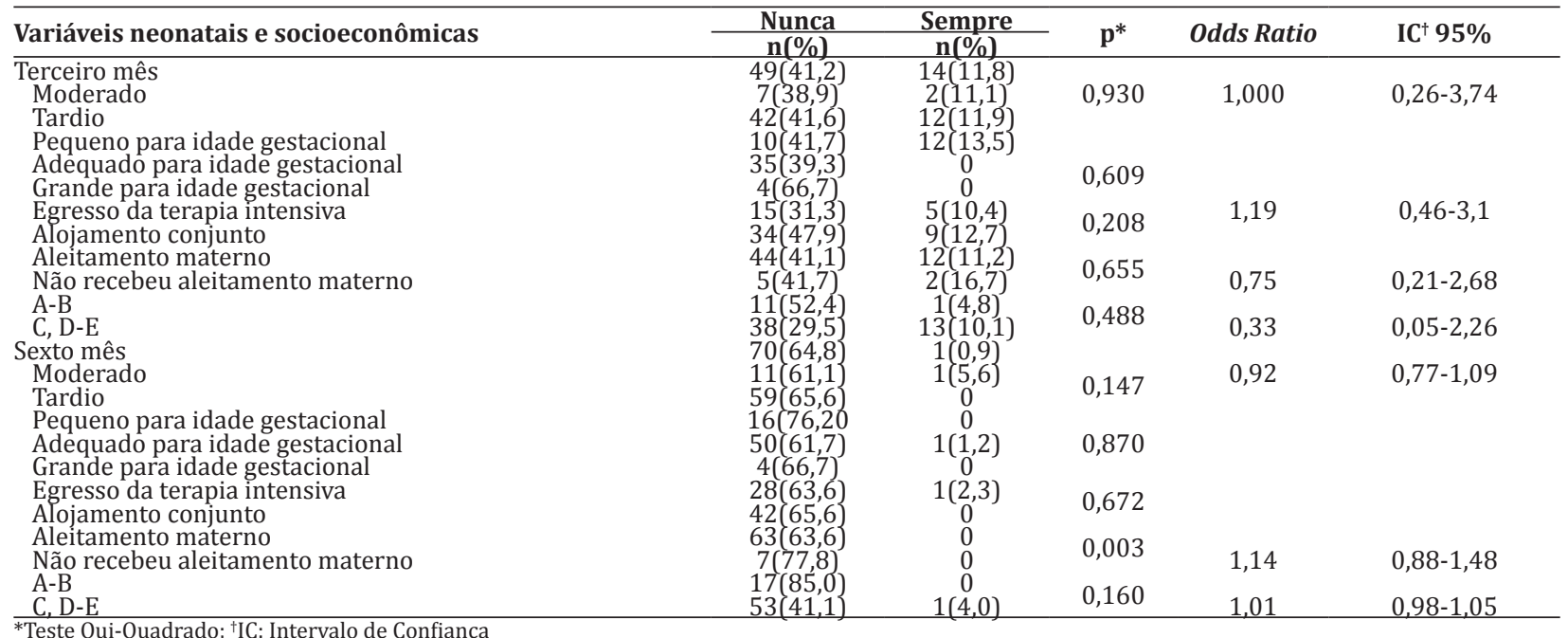


Na análise das variáveis neonatais e socioeconômicas, não se percebeu diferença significativa entre a intensidade da cólica no terceiro mês de vida. No terceiro mês, $16,7 \%$ dos bebês que não receberam aleitamento materno apresentaram cólica sempre, comparados aos $11,2 \%$ daqueles que estavam em aleitamento materno exclusivo. Porém, no sexto mês de vida, houve diferença significativa entre a intensidade da cólica. Evidencia-se que, no sexto mês, 63,6\% dos bebês que receberam aleitamento materno exclusivo, nunca apresentavam cólica, comparados aos $77,8 \%$ daqueles que estavam em aleitamento materno. Contudo, não se observou diferença significativa entre a intensidade da cólica no terceiro mês de vida dos bebês pequenos para idade gestacional, os quais apresentaram $13,5 \%$ de intensidade sempre, comparados aos bebês adequados para idade gestacional e grandes para idade gestacional que não apresentaram o agravo no período.

Sob a análise dos três agravos, destaca-se que a cólica foi mais frequente no terceiro mês, quando comparada ao sexto mês. Os demais agravos, vômito e diarreia, apresentaram percentuais semelhantes, nos dois períodos avaliados ( $3^{\circ}$ e $12^{\circ}$ mês de vida), o que pode representar tendência dessas complicações ao longo do primeiro ano da vida, quanto à intensidade.

\section{Discussão}

Consideram-se limitações deste estudo a coleta dos achados clínicos, por meio da percepção materna ou principal cuidador, contudo, entende-se que a repercussão do agravo é vivida pela família/cuidador e esta é quem deve considerar o quanto intenso o agravo repercute no contexto familiar e da criança. A percepção desta irá guiar a procura dos serviços e formação da rede de atenção à saúde. Outra fragilidade, como os demais estudos longitudinais, refere-se às perdas de acompanhamento, por desistência ou ausência de contato.

Todavia, este estudo aborda aspectos relevante acerca das condições de saúde de população cada vez mais prevalente e fisiologicamente imatura. A identificação da prevalência e dos fatores de risco permite a organização dos serviços de puericultura e seguimento, na perspectiva dos agravos, visto que as afecções gastrointestinais vômito, cólica e diarreia são as principais manifestações clínicas no primeiro ano de vida. Esses sinais podem estar associados à fisiologia digestiva normal ou do desenvolvimento específico desse sistema, no primeiro ano de vida. Ao relacionar o recém-nascido prematuro à imaturidade do sistema digestivo e alimentação, é possível que essas crianças apresentem repercussões negativas, como o vômito ${ }^{(10)}$.

Neste estudo, para população de prematuros tardios e moderados, o vômito foi o mais prevalente no terceiro mês de vida, quando comparado ao final do primeiro ano de vida. Estudo mostra que o vômito nos primeiros meses de vida está associado ao refluxo gastresofágico e que, em maioria, caracteriza-se de forma fisiológica e mais frequente no início da vida ${ }^{(12)}$. Trata-se de afecção comum na infância, tem prevalência de aproximadamente $30,0 \%$ dos lactentes a termo, no primeiro ano de vida, e torna-se mais frequente na população de nascidos prematuros ${ }^{(9-10)}$. Assim, podese inferir que o vômito em crianças prematuras segue padrões da população dos demais prematuros, cuja prevalência é maior, quando comparadas aos a termos.

0 vômito por refluxo gastresofágico, geralmente, melhora com a idade, menos de 5,0\% das crianças com vômito ou regurgitação, na infância, continuam a apresentar sintomas após os 12 meses de idade. Isso ocorre devido à combinação de crescimento no comprimento do esôfago, postura mais ereta, aumento dos tônus do esfíncter esofágico inferior e dieta mais sóli$\mathrm{da}^{(13)}$.

Os principais fatores, associados ao vômito, incluem o alto volume de leite ingerido, postura e imaturidade funcional do esfíncter esofágico inferior, este último se encontra fortemente relacionado à prematuridade. Constata-se que nascidos prematuros tardios e moderados são mais vulneráveis a apresentar vômito, devido ao fator biológico, à alta precoce hospitalar 
e às demais repercussões clínica da prematuridade ${ }^{(9)}$. Estudo com recém-nascidos prematuros destaca prevalência de 2,0 a 6,0\% na população de prematuros e considera risco, fatores como baixo peso, idade gestacional menor de 34 semanas e tempo maior de 30 dias de internação ${ }^{(14)}$.

0 vômito pode também comprometer o crescimento e o desenvolvimento da criança, sendo apontado como causador de estresse para pais e cuidadores. Quando essas crianças ainda estão no hospital, os cuidados para prevenir e tratar as causas do vômito são realizados por profissionais e direcionados à alimentação. Contudo, na ocasião da alta, esses cuidados são realizados por familiares e podem também ser bem apropriados, na prevenção de aspirações e asfixias ${ }^{(15)}$.

A diarreia, por não estar associada a condições de imaturidade fisiológica, tão pouco, a processos de adaptação fisiológica, manteve intensidade frequente ao longo das avaliações dos terceiro e $12^{\circ}$ meses de vida neste estudo. Considera-se que se apresenta mais intensa nos primeiros meses de vida, quando comparados aos demais. 0 adoecimento das crianças menores de um ano de vida, por diarreia, pode estar relacionado ao ponto de vista biológico, agravados com fatores como a prematuridade, o baixo peso ao nascer e a malformação congênita ${ }^{(16)}$.

Lactentes que não estavam em aleitamento materno exclusivo apresentaram maior intensidade de diarreia na avalição do terceiro mês ( $p=0,012)$, bem como aqueles classificados em classes C, D-E apresentaram maior intensidade do agravo ao final do primeiro ano de vida $(p=0,043)$. Esse achado corrobora com estudo realizado no interior do Ceará, o qual revelou que quanto menor a renda familiar, menor a autoeficácia materna para prevenir a diarreia, especialmente para lactentes menores de um ano e maior risco desse agravo $^{(17) .}$

0 aleitamento materno é uma intervenção fundamental na proteção contra a diarreia aguda. Crianças menores de seis meses que estavam em aleitamento materno exclusivo, tiveram menos episódios de diarreia, dado que confirma a medida, pois devido à introdução de outros alimentos à dieta dos bebês e diminuição do aleitamento exclusivo, constatou-se incremento de episódios de diarreia infantil ${ }^{(16)}$.

Ainda, como agravo mais frequente nos primeiros meses de vida, a cólica pode estar relacionada à imaturidade fisiológica, principalmente nos casos mais intensos de prematuros tardios e moderados. Orientar aos pais que a cólica pode ser um fenômeno natural do desenvolvimento gastrointestinal da criança, auxilia-os em medos e angústias, com vistas a diminuir a ansiedade frente ao choro ${ }^{(18)}$.

Quanto aos fatores associados à intensidade da cólica, nos terceiro e sexto meses de vida, destaca-se que a alimentação foi considerada fator predisponente. No que tange aos fatores associados à cólica, estudo afirma não existir diferença entre sexo ou tipo de dieta, dado que difere do apresentado por este estudo. Essa disparidade pode estar associada à diferença de população entre os estudos, não considerando a prematuridade como viés. Contudo, as evidências destacam que manter o aleitamento materno permite o controle dessa manifestação(10).

As estratégias de ações comportamentais entre pais e filhos podem auxiliar no enfrentamento desses sintomas, e a cólica pode ser encarada de forma diferente, na percepção de cada família. 0 suporte social oferecido a mãe pode ser importante fator para diminuição dos episódios de cólica. Em outro estudo, constatou-se que bebês de mães que recebiam apoio afetivo apresentaram menos sintomas de cólicas ${ }^{(19)}$.

\section{Conclusão}

Para prematuros tardios e moderados, são mais intensos, nos três primeiros meses de vida, as afecções vômito e cólica, e a diarreia se mantém constante ao longo do primeiro ano de vida. Não se identificaram fatores neonatais e socioeconômicos associados ao desenvolvimento de vômito. Contudo, para as afecções diarreia e cólica, destaca-se que prematuros que não estão em aleitamento materno exclusivo, apresentam maior intensidade de cólica e diarreia. Também 
é mais frequente, ao final do primeiro ano de vida, o desenvolvimento de diarreia, naqueles pertencentes à classes socioeconômicas C, D-E.

\section{Colaborações}

Jantsch LB colaborou na concepção e projeto, análise e interpretação dos dados, redação do artigo, revisão crítica relevante do conteúdo intelectual e aprovação final da versão a ser publicada. Barzotto VS e Silva EB contribuíram na análise e interpretação dos dados, redação do artigo, revisão crítica relevante do conteúdo intelectual e aprovação final da versão a ser publicada.

\section{Referências}

1. World Health Organization. Preterm birth [Internet]. 2015 [cited Jan 12, 2020]. Available from: http://www.who.int/mediacentre/factsheets/ fs363/en/

2. Delnord M, Zeitlin J. Epidemiology of late preterm and early term births - an international perspective. Seminars Fetal Neonatal Med. 2019; 24(1):310. doi: doi.org/10.1016/j.siny.2018.09.001

3. Vanin LK, Zatti H, Soncini T, Nunes RD, Siqueira LBS. Maternal and fetal risk factors associated with late preterm infants. Rev Paul Pediatr. 2020; 38:e2018.136. doi: http://dx.doi. org/10.1590/1984-0462/2020/38/2018136

4. Camilleri M, Park SY, Scarpato E, Staiano A. Exploring hypotheses and rationale for causes of infantile colic. Neurogastroenterol Motil. 2017; 29(2):e12943. doi: doi.org/10.1111/nmo.12943

5. Flake ZA, Linn BS, Hornecker JR. Pratical selection of antiemetics in the ambulatory setting. Am Fam Physician [Internet]. 2015 [cited Jan 19, 2020]; 91(5):293-6. Available from: https://www.ncbi. nlm.nih.gov/pubmed/25822385

6. Baird DC, Harker DJ, Karmes AS. Diagnosis and treatment of gastroesophageal reflux in infants and children. Am Fam Physician [Internet]. 2015 [cited Jan 19, 2020]; 92(8):705-14. Available from: https://www.ncbi.nlm.nih.gov/ pubmed/26554410
7. Solé D, Silva LR, Cocco RR, Ferreira CT, Sarni RO, Oliveira LC, et al. Diagnóstico, tratamento e prevenção. Documento conjunto elaborado pela Sociedade Brasileira de Pediatria e Associação Brasileira de Alergia e Imunologia. Braz J Allergy Immunol [Internet]. 2018 [cited Jan 19, 2020];2(1):39-82. Available from: http://aaaiasbai.org.br/detalhe_artigo.asp?id=865

8. Tovar JA. Gastroesophageal reflux in newborns and premature infants. Rickham's Neonatal Surgery. 2018; 577-93. doi: dx.doi.org/10.1007/9781-4471-4721-3_25

9. Tovar JA. Gastroesophageal reflux in the first year of life. Neonatal Surgery. 2019; 209-23. doi: http://dx.doi.org/10.1007/978-3-319-935348_15

10. Morais MB. Signs and symptoms associated with digestive tract development. J Pediatr. 2016; 92(3Suppl1):46-56. doi: https://doi. org/10.1016/j.jped.2016.02.008

11. Fekkes M, Theunissen N, Brugman E. Veen EGH, Verrips HM, Koopman T, et al. Development and psychometric evaluation of the TAPQOL: A healthrelated quality of life instrument for 1-5-yearold children. Qual Life Res. 2000; 9:961-72.doi: https://doi.org/10.1023/A:1008981603178

12. Kolimarala V, Beattie RM, BatraA. Gastro-oesophageal reflux in infancy. Paediatr Child Health. 2019; 29(9):377-83. doi: https://doi.org/10.1016/j. paed.2019.06.002

13. Leung AK, Hon KL. Gastroesophageal reflux in children: an updated review. Drugs Context. 2019; 8:212591. doi: http://dx.doi.org/10.7573/ dic. 212591

14. Beleza LO, Ribeiro LM, Paula RAP, Guarda LEDA, Vieira GB, Costa KSF. Profile of at-risk newborns attended by nurses in outpatient follow-up clinic: a retrospective cohort study. Rev Latino-Am Enfermagem. 2019; 27: e3113. doi: https://doi. org/10.1590/1518-8345.2301.3113

15. Pagliaro CL, Bühler KEB, Ibidi SM, Limongi SCO. Dificuldades de transição alimentar em crianças prematuras: revisão crítica de literatura. J Pediatr. 2016; 92(1):7-14. https://doi.org/10.1016/j. jped.2015.05.004 
16. Vasconcelos MJOB, Rissin A, Figueiroa JN, Lira PIC, Batista FM. Factors associated with diarrhea in children under five years old in the state of Pernambuco, according to surveys conducted in 1997 and 2006. Rev Saúde Pública. 2018; 52:48. doi: http://dx.doi.org/10.11606/s15188787.2018052016094

17. Oliveira RKL, Oliveira BSB, Bezerra JC, Silva MJN, Melo FMS, Joventino ES Influence of socioeconomic conditions and maternal knowledge in self-effectiveness for prevention of childhood diarrhea. Esc Anna Nery. 2017; 21(4):e20160361. doi:https://doi.org/10.1590/2177-9465ean-2016-0361
18. Marcon ACC, Vieira MC, Morais MB. Conhecimentos do pediatra sobre o manejo do lactente que chora excessivamente nos primeiros meses de vida. Rev Paul Pediatr. 2014; 32(2):187-92. doi: http:// dx.doi.org/10.1590/0103-0582201432218713

19. Alexander CP, Zhu J, Paul IM, Kjerulff KH. Fathers make a difference: positive relationships with mother and baby in relation to infant colic. Child Care Health Dev. 2018; 43(5):687-96. doi: http:// dx.doi.org/10.1111/cch.12445

\section{(cc) BY}

Este é um artigo de acesso aberto distribuído sob os termos da Licença Creative Commons 